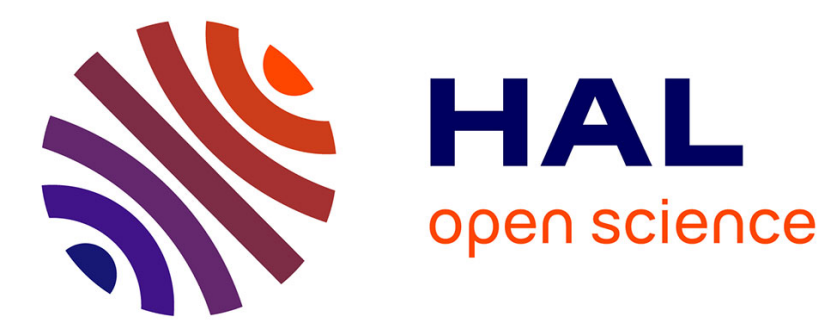

\title{
A sampled spur free fractional frequency synthesizer and its noise analysis
}

\author{
Marc Houdebine, Sébastien Dedieu, Olivier Sename, Mazen Alamir
}

\section{To cite this version:}

Marc Houdebine, Sébastien Dedieu, Olivier Sename, Mazen Alamir. A sampled spur free fractional frequency synthesizer and its noise analysis. 2006. hal-00086709

\section{HAL Id: hal-00086709 \\ https://hal.science/hal-00086709}

Submitted on 19 Jul 2006

HAL is a multi-disciplinary open access archive for the deposit and dissemination of scientific research documents, whether they are published or not. The documents may come from teaching and research institutions in France or abroad, or from public or private research centers.
L'archive ouverte pluridisciplinaire HAL, est destinée au dépôt et à la diffusion de documents scientifiques de niveau recherche, publiés ou non, émanant des établissements d'enseignement et de recherche français ou étrangers, des laboratoires publics ou privés. 


\section{A sampled spur free fractional frequency synthesizer and its noise analysis}

\author{
Marc Houdebine, Sébastien Dedieu \\ ST Microelectronics Crolles, \\ 850 rue Jean Monnet, \\ 38926 Crolles Cedex, FRANCE \\ Email: \{Marc.Houdebine, Sebastien.Dedieu\}@st.com
}

\author{
Olivier Sename, Mazen Alamir \\ Laboratoire d'Automatique de Grenoble, \\ UMR CNRS-INPG-UJF 5528, ENSIEG-BP 46, \\ 38402 Saint Martin d'Hères Cedex, FRANCE. \\ Email: $\{0 l i v i e r . S e n a m e$, Mazen.Alamir\}@inpg.fr
}

\begin{abstract}
This paper presents a new fractional frequency synthesizer architecture and its noise analysis model. The proposed analysis model takes into account the sampled behavior of the PLL. In order to validate this study, measurement results illustrate the output frequency purity and the reliability of the model.
\end{abstract}

\section{INTRODUCTION}

Widely used in modern electronics, local oscillators are based on Phase Locked Loop architectures (PLL) by locking a tunable oscillator (VCO) to an accurate frequency source as a crystal oscillator. This reference clock gives rhythm to the Voltage Controlled Oscillator (VCO) input voltage refreshing and induces parasitic rays to the output phase noise spectrum. Because the frequency spacing between these parasitic rays called "spurious" and the carrier frequency is equal to the reference clock frequency, the reference clock is chosen as high as possible. Moreover, by increasing the reference clock frequency, the loop bandwidth can be larger which decreases the settling time. Another benefit is the outputreference frequency ratio diminishing which reduces the output noise contribution of the reference clock. All these advantages induce to increase the reference clock frequency as high as possible, but to meet the output frequency step lower than the reference frequency value, the output-reference frequency ratio have to be real and not only integer.

Because low noise frequency dividers are comparable to counters, they can only make integer division. To make fractional division, classical PLLs uses several dividers on the feedback path switched according to the desired fractional frequency ratio degrading the output spectra purity by adding other spurious tones due to "quantization noise" [1].

The structure presented in this paper is a spuriousless fractional RF frequency synthesizer taking advantages of a sampled working to get free of the limiting quantization noise.

To study the noise performances of the loop, linear continuous models are mostly used providing a loop bandwidth much below than the sampling frequency fixed by the reference clock [2]. Some models consider the sampling as a delay [3], [5]. It has also been presented a mixed $z-s$ model in [4] close to classical models. In this work, we propose an appropriate discrete time model taking the switch aperture time into account.
The content of this paper is as follows. The proposed spur free fractional frequency synthesizer architecture is presented in section II. Section III develops the appropriate noise model taking the sampled behavior into account. Some measurement results are depicted in section IV.

\section{THE SPUR FREE FRACTIONAL FREQUENCY SYNTHESIZER}

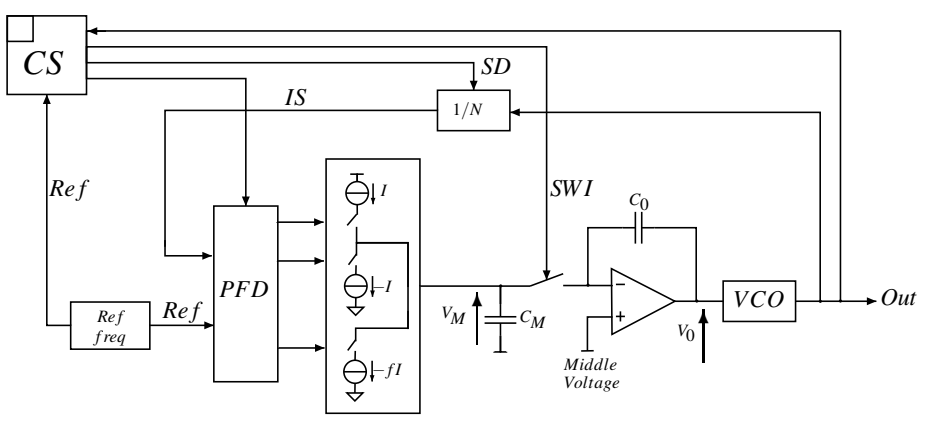

Fig. 1. The Spur free Fractional Frequency Synthesizer architecture

The proposed fractional frequency synthesizer architecture is shown in Fig. 1. The removal of quantization noise is due to the system working by two time cycles: first the output frequency measurement followed by the VCO input control voltage correction. During the output frequency measurement, charges are injected into the measurement capacitor $V_{M}$. In order to avoid disturbing the output frequency during the measurement, the signal SWI opens the switch to isolate the VCO from the measurement capacitor $C_{M}$. The VCO input control voltage $V_{0}$ is kept constant by the capacitor $C_{0}$. As shown in Fig. 2, when the output frequency measurement is done, the phase displacement between the reference clock and the output is memorized into capacitor $C_{M}$. The input voltage correction is then possible by closing the switch starting the charge transfer from capacitor $C_{M}$ to $C_{0}$.

\section{A. The output measurement method}

Let $N$ be the integer part of the frequency ratio and $f$ the corresponding fractional part:

$$
F_{\text {out }}=(N+f) F_{\text {ref }} \quad\left\{\begin{array}{l}
N \in \mathbb{N} \\
0 \leqslant f \leqslant 1
\end{array}\right.
$$




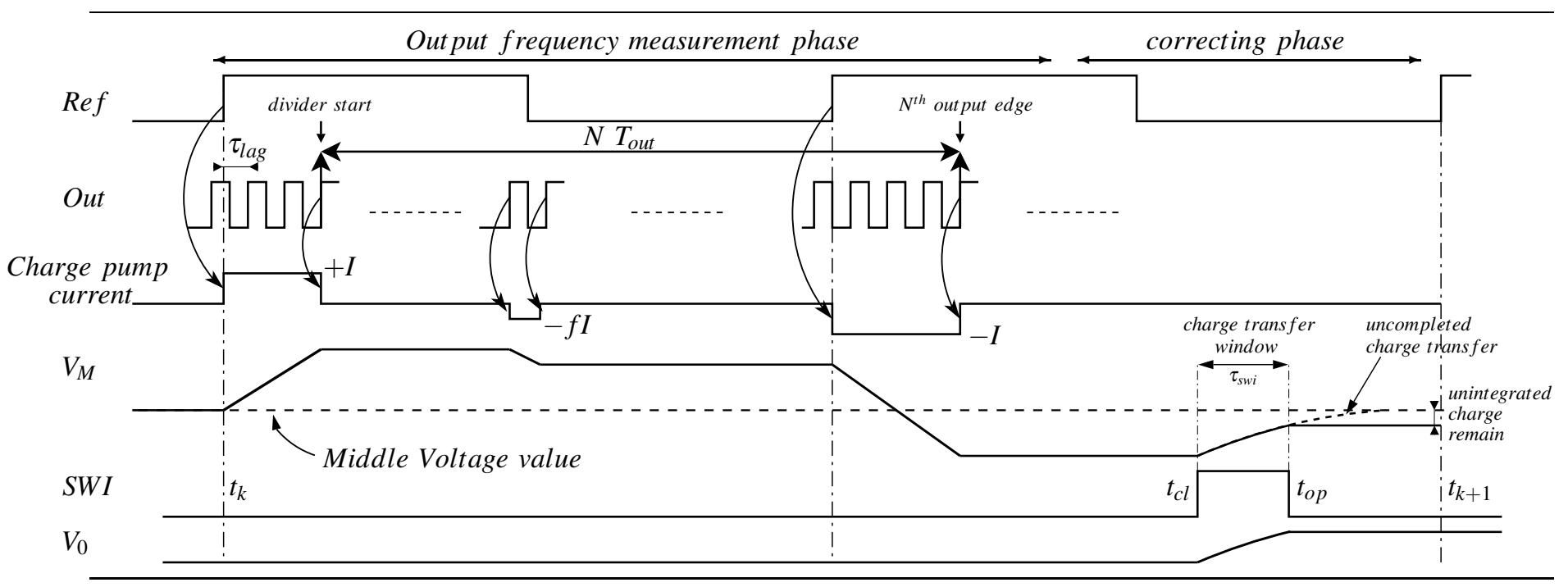

Fig. 2. Chronogram of the sampled Frequency Synthesizer architecture in an unlocked case.

When the system is locked, a cyclic phase displacement $\tau_{\text {lag }}$ is due to the fractional part since $T_{\text {ref }}=(N+f) T_{\text {out }}$. In order to be insensitive to this natural phase displacement, two opposite currents are injected from the reference phase to the output phase. To avoid any dead zone, the first current is injected for more than 2 output periods. The last current injection will stop at the $N^{\text {th }}$ output rising edge from the divider start (see Fig.2). The fractional part is added to these currents during one output period and in the end of measurement, charges stored in a lock case are equal to:

$\Delta Q=\left(\tau_{\text {lag }}+2 T_{\text {out }}\right) I-f I T_{\text {out }}-I\left(\tau_{\text {lag }}+(1-f) T_{\text {out }}+T_{\text {out }}\right)=0$.

\section{B. The correcting charge transfer}

In Fig.2, an unlocked case is voluntary shown in order to illustrate the charge transfer through the switch window. As detailed in Fig.3, the resistor $R$ makes possible the loop bandwidth setting. On the other hand, the charge transfer is slowed down. For practical reasons, this resistor is placed before the switch in order to have a small contribution to the PLL output noise. For noise reduction, the amplifier is reduced to a simple MOS and the Middle Voltage is fixed by its $V_{g s}$. This Middle Voltage corresponds to the mean value of the measurement voltage $V_{M}$ at the charge pump output. Because charge pump sink and source currents are

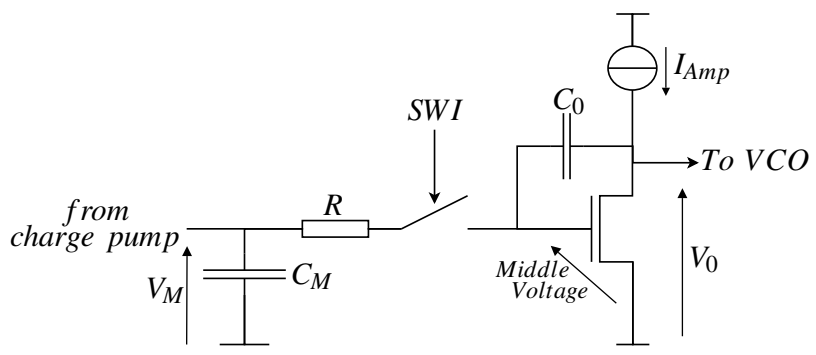

Fig. 3. Charge transfert circuit details best equivalent $(|+I|=|-I|)$ when its output bias voltage is kept close to half the supply voltage, the MOS transistor is dimensioned to set its $V_{g s}$ at half the supply voltage.

So, for whole charge transfer, $V_{M}$ comes back to its mean value. But when it is not the case as shown in Fig.2, some charges remain in $C_{M}$ and will have to be discharged for the next time added to the next accumulated measurement charges.

This bring us to the necessity of an appropriate noise model. For more details, please see [6], and for further information about the system nonlinearity, see [7].

\section{AN APPROPRIATE MODEL TO SAMPLE DATA SYSTEMS}

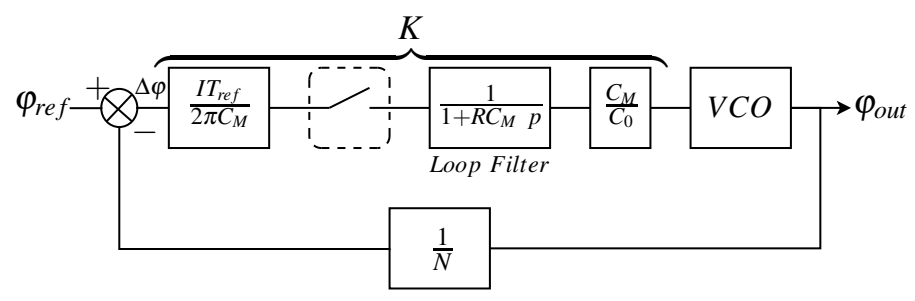

Fig. 4. Small signal Model establishing

As shown in the previous section, the switch window has to be considered in small signal models.

Because the voltage $V_{0}$ is kept constant by capacitors $C_{0}$ when the switch is open (see Fig.2), its value at the switch closing instant $t_{c l}$ is equal to its value at instant $t_{k}$. Then, at the switch opening instant $t_{o p}$, its value doesn't change until the instant $t_{k+1}$. In view of this, we can consider only the instant $t_{k}$ and $t_{k+1}$.

As well as for $V_{0}$, the value of $V_{M}$ doesn't change from instant $t_{o p}$ to the instant $t_{k+1}$. Because the measurement charges are injected into $C_{M}$ while the switch is open, regarding from $V_{0}$, $V_{M}$ value has changed instantaneously at the instant $t_{k}$. In fine, at the sight of the VCO control voltage, instant $t_{k}$ and $t_{k+1}$ can be considered as respectively the switch opening 
and closing instant, and the sampling period is equivalent to the closed switch window $\tau_{s w i}$. On the other hand, during the switch opening, the charge pump current is integrated for the time difference $\Delta t=\Delta \varphi \frac{T_{r e f}}{2 \pi}$ and $V_{M}$ results from these charges integration into $C_{M}$ :

$$
V_{M}=\frac{I T_{r e f}}{2 \pi C_{M}} \Delta \varphi
$$

Then, the expression of $K$ (Fig. 4) can be found by the exact discretization method [8]:

$$
K(z)=\frac{I T_{r e f}}{2 \pi C_{M}} \frac{C_{M}}{C_{0}}\left(1-z^{-1}\right) Z\left\{\left.\mathcal{L}^{-1}\left[\frac{1}{s\left(1+R C_{M} s\right)}\right]\right|_{\tau_{s w i}}\right\}
$$

Because the MOS grid (Fig. 3) voltage doesn't significantly change, the effective components involved in the filtering are only $R$ and $C_{M}$. Charges are then transferred from $C_{M}$ to $C_{0}$ such that the charge conservation implies: $Q=C_{M} V_{M}=C_{0} V_{0}$. Equation (3) leads to:

$$
K(z)=\frac{I T_{r e f}}{2 \pi C_{0}} \frac{\left(1-e^{-\frac{\tau_{s w i}}{R C_{M}}}\right)}{\left(z-e^{-\frac{\tau_{s w i}}{R C_{M}}}\right)}
$$

Likewise, the equivalent VCO discrete time model is:

$$
G(z)=2 \pi K_{o} \frac{2 T_{r e f}}{z-1}
$$

It is worth noting that the presented frequency synthesizer only needs one divider on the feedback path such that the corresponding model is a simple gain equal to the opposite of the frequency ratio integer part.

This approach which takes the switch opening and closing time into account will now be compared with measurement results.

\section{Measurement Results}

A fully integrated test chip was designed in technology CMOS 120nm. The circuit layout uses the 6 metal levels and capacitors $C_{M}$ and $C_{0}$ are Metal On Metal capacitors (MOM) from metal 2 to metal 5, no specific option as inter metal thin

\begin{tabular}{|c|c|}
\hline VCO & $0.211 \mathrm{~mm}^{2}$ \\
\hline control loop & $0.162 \mathrm{~mm}^{2}$ \\
\hline Total & $0.39 \mathrm{~mm}^{2}$ \\
\hline
\end{tabular}

a. Silicon Area

\begin{tabular}{|l|r|}
\hline VCO+buffer & $48.5 \mathrm{~mA}$ \\
\hline Analog part & $3.1 \mathrm{~mA}$ \\
\hline Digital part & $1.4 \mathrm{~mA}$ \\
\hline Total & $53 \mathrm{~mA}$ \\
\hline
\end{tabular}

b. Current consumption

\begin{tabular}{|c|c|}
\hline$F_{\text {ref }}$ & $27 \mathrm{MHz}$ \\
\hline$F_{\text {out }}$ & $1.2 \mathrm{GHz}-1.75 \mathrm{GHz}$ \\
\hline$\tau_{\text {swi }}$ & $1.5 \mathrm{~ns}$ \\
\hline
\end{tabular}

c. Time/Frequency values

\begin{tabular}{|c|c|}
\hline$R$ & $20 \mathrm{k} \Omega$ \\
\hline$C_{M}$ & $20 \mathrm{pF}$ \\
\hline$C_{0}$ & $20 \mathrm{pF}$ \\
\hline$K_{0}$ & $100 \mathrm{MHz} / \mathrm{V}$ \\
\hline$I_{\text {chpp }}$ & $200 \mu \mathrm{A}$ \\
\hline
\end{tabular}

d. parameter values

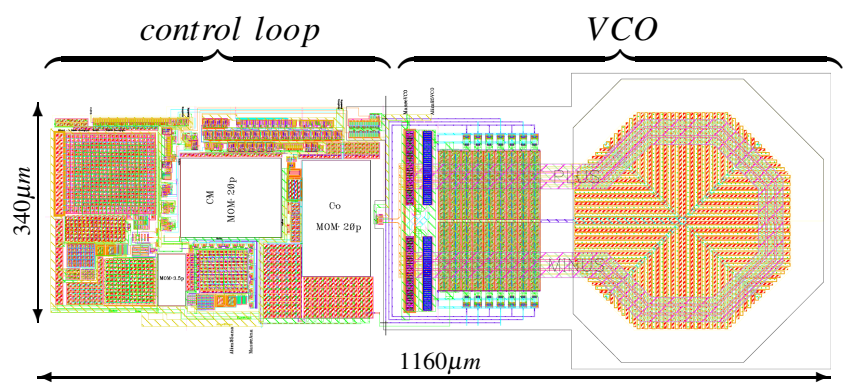

Fig. 5. view of circuit layout

oxide capacitor (MIM) was used. The circuit characteristics are reported in table I. The output frequency range is from $1.2 \mathrm{GHz}$ to $1.75 \mathrm{GHz}$ by $210 \mathrm{kHz}$ steps. The reference quartz frequency is $27 \mathrm{MHz}$.

As we can see in Fig. 6, there are still one unavoidable spur owing to the VCO tuning node refreshing at each sampled time. The reference frequency is chosen as high as possible to decrease the sample time in order to push aside these spurs from the carrier. Because two reference clock periods are needed to execute one sample time, the carry-spur spacing is equal to $F_{r e f} / 2=13.5 \mathrm{MHz}$. Added to these spurs, additional

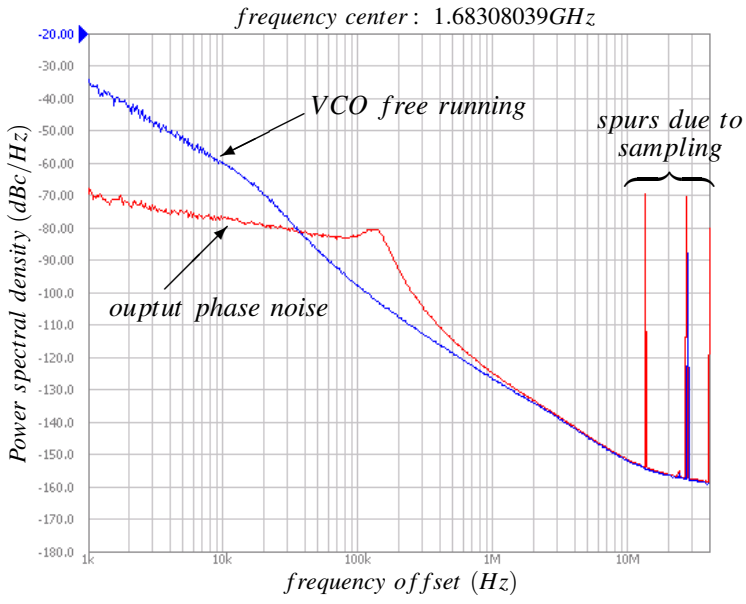

Fig. 6. output Phase Noise measurement (Agilent E5052A)

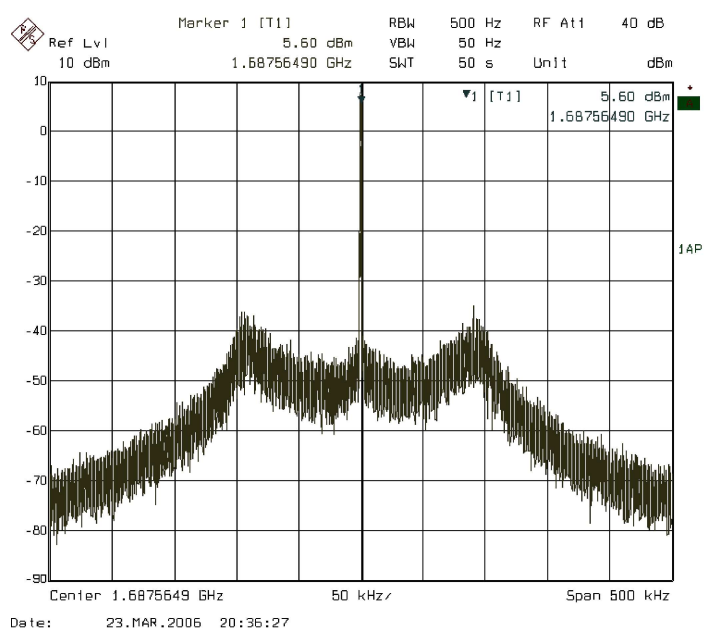

Fig. 7. Spectrum analyser measurement 


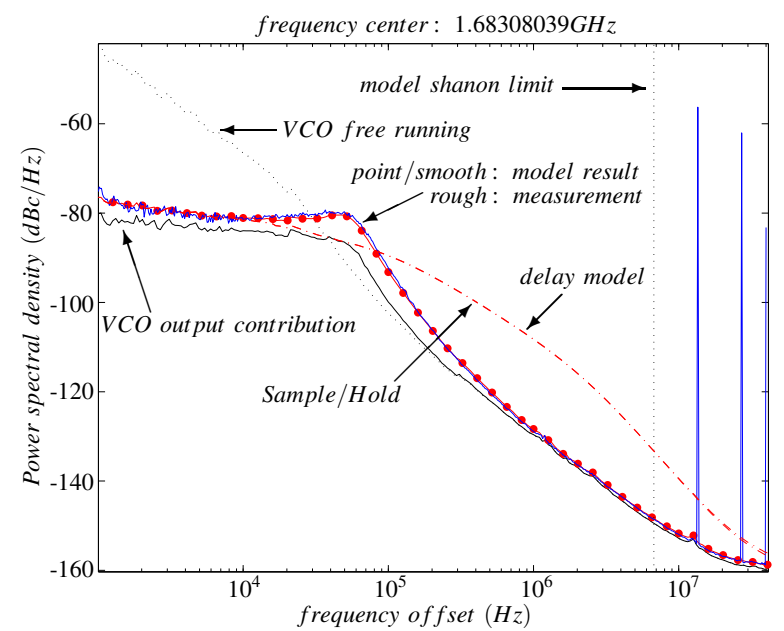

Fig. 8. model/measurement corroboration with $I_{\text {chpp }}=50 u A$

rays are due to clock feedthrough of the sampling switch. Indeed, because the switch is a MOS transistor, when its grid command SWI changes, its parasitic capacitors values changes inducing a small parasitic charge injection. This charges injected or suppressed at instants $t_{c l}$ and $t_{o p}$ are integrated in $C_{0}$ and creates periodic disturbances at the VCO input control voltage as detailed in [4]. As shown in [4], the worst case on the switch opening command SWI is a $50 \%$ duty cycle.

Fig. 6 shows the PLL output phase noise measured with the Agilent E5052A signal source analyser. It represents the offset frequency from the carrier shown in Fig. 7. The center frequency is such that the output-reference frequency ratio is equal to: $N+f=62.336$. Spurs due to the sampling clearly appear at one $(13.5 \mathrm{MHz})$, at twice $(27 \mathrm{MHz})$ and three times $(40.5 \mathrm{MHz})$ the sampled frequency. In spite of these well known and unavoidable spurs, there is no added spurious due to the fractional part.

Fig. 8 and 9 show the presented model result overwritten on measurement results with a charge pump current of respectively $50 \mu \mathrm{A}$ and $200 \mu \mathrm{A}$. Dashed-doted lines also show the output noise calculated by considering the sampling as a delay $e^{-2 T_{r e f} s}$ (delay model) or by establishing a sampled and hold model with a frequency sampling at $2 T_{\text {ref }}$. Our model is more suitable because it takes the switch opening and closing time into account especially when the closing time is much lower than the opening time.

The model shanon limit shown in dashed line on Fig. 8 and 9 represents the model maximum frequency validity. Because this frequency at half the sampling period is much larger than the PLL bandwidth, no noise information is hidden. Indeed, as it is clearly shown, the VCO output noise contribution is high-pass filtered by the loop. For frequencies over $1 \mathrm{MHz}$, the VCO is the main output contributor. So, over the model shanon limit, the PLL output phase noise is equal to the VCO phase noise.

This test chip target was to validate this new PLL architecture and its study, so the circuit performances were not optimum but it will be so in the next circuit.

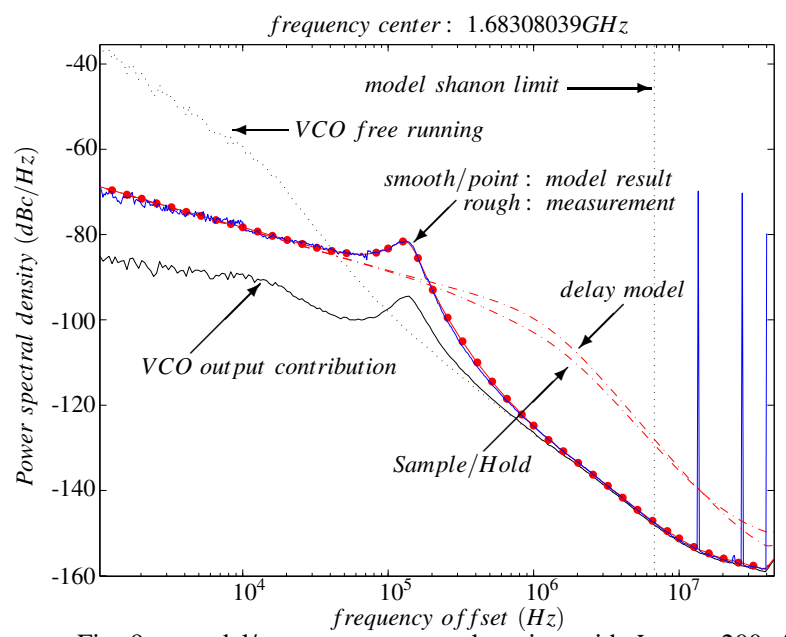

Fig. 9. model/measurement corroboration with $I_{\text {chpp }}=200 u A$

\section{CONCLUSION}

A fully integrated on silicon RF frequency synthesizer has been presented. It has been demonstrated that the sampled working is the solution to avoid any fractional spur.

Because the switch aperture is much lower than the reference clock period, classical continuous models are ineffective for noise analysis. Then, a discrete time model was established to study this PLL output phase noise profile. The sampled model reliability as well as this new PLL architecture has been validated with a chip demonstrator.

\section{ACKNOWLEDGMENTS}

The authors gratefully acknowledge Gerald Provins (STMicroelectronics) for his helpful contribution in the VCO design and assembling.

\section{REFERENCES}

[1] S.E. Meninger and M.H. Perrott, "Bandwidth extension of low noise fractional- $N$ Synthesizers", IEEE Radio Frequency Integrated Circuits Symposium-RFIC, p.211-214, June 2005.

[2] J.A. Crawford, "Frequency Synthesizer Design Handbook", ArtechHouse Boston.London, chapter 4, Appendix 4A, 1994.

[3] Y. Tang, M. Ismail and S. Bibyk, "A new fast-settling gearshift adaptative PLL to extend loop bandwidth enhancement in frequency synthesizers", IEEE International Symposium on Circuits And Systems-ISCAS, vol. 4, May 2002.

[4] B. Zhang, Phillip E. Allen and Jeff M. Huard, "A Fast Switching PLL Frequency Synthesizer With an On-Chip Passive Discrete-Time Loop Filter in 0.25- $\mathrm{mm}$ CMOS", IEEE Journal of Solid-State Circuits, vol. 38, No. 6, June 2003.

[5] M. Cassia, P. Shah and E. Bruun, "Analytical Model and Behavioral Simulation Approach for a $\Sigma \Delta$ Fractional-N Synthesizer Employing a Sample-Hold Element", IEEE Transactions on Circuits And Systems-II: Analog And Digital Signal Processing, vol. 50, No. 11, November 2003.

[6] M. Houdebine and S.Dedieu, "Method and Device for generating a signal by fractional frequency locked loop.”, STM patent pending.

[7] M. Houdebine, S. Dedieu, M. Alamir and O. Sename, "A New Fractional Frequency Synthesizer Architecture With Stability and Robustness Analysis”, Proceedings of the IFAC World Congress, Prague, Czech Republic, Jul. 2005.

[8] K. Astrom and B. Wittenmark, "Computer Controlled Systems, Theory and design”, prentice hall, 1997. 